\title{
Diverse Immunoprofile of Ductal Adenocarcinoma of the Prostate with an Emphasis on the Prognostic Factors
}

\author{
Se Un Jeong \\ Anuja Kashikar Kekatpure \\ Ja-Min Park ${ }^{1}$. Minkyu Han ${ }^{2}$ \\ Hee Sang Hwang · Hui Jeong Jeong \\ Heounjeong Go · Yong Mee Cho \\ Department of Pathology, ${ }^{1}$ Asan Institute for Life \\ Sciences, ${ }^{2}$ Department of Clinical Epidemiology \\ and Biostatistics, Asan Medical Center, University \\ of Ulsan College of Medicine, Seoul, Korea \\ Received: February 28, 2017 \\ Revised: May 13, 2017 \\ Accepted: June 2, 2017 \\ Corresponding Author \\ Yong Mee Cho, MD, PhD \\ Department of Pathology, University of Ulsan \\ College of Medicine, Asan Medical Center, \\ 88 Olympic-ro 43-gil, Songpa-gu, Seoul 05505, \\ Korea \\ Tel: +82-2-3010-5965 \\ Fax: +82-2-3010-7898 \\ E-mail:yongcho@amc.seoul.kr
}

\begin{abstract}
Background: Ductal adenocarcinoma (DAC) of the prostate is an uncommon histologic subtype whose prognostic factors and immunoprofile have not been fully defined. Methods: To define its prognostic factors and immunoprofile, the clinicopathological features, including biochemical recurrence $(\mathrm{BCR})$, of 61 cases of DAC were analyzed. Immunohistochemistry was performed on tissue microarray constructs to assess the expression of prostate cancer-related and mammalian target of rapamycin (mTOR) signaling-related proteins. Results: During the median follow-up period of 19.3 months, BCR occurred in 26 cases (42.6\%). DAC demonstrated a wide expression range of prostate cancer-related proteins, including nine cases (14.8\%) that were totally negative for pan-cytokeratin (PanCK) immunostaining. The mTOR signaling-related proteins also showed diverse expression. On univariate analysis, BCR was associated with high preoperative serum levels of prostate-specific antigen (PSA), large tumor volume, predominant ductal component, high Gleason score (GS), comedo-necrosis, high tumor stage (pT), lymphovascular invasion, and positive surgical margin. High expressions of phospho-mTOR (p-mTOR) as well as low expressions of PSA, phospho-S6 ribosomal protein (pS6) and PanCK were associated with BCR. On multivariable analysis, GS, pT, and immunohistochemical expressions of PanCK and p-mTOR remained independent prognostic factors for BCR. Conclusions: These results suggest GS, pT, and immunohistochemical expressions of PanCK and p-mTOR as independent prognostic factors for BCR in DAC. Since DAC showed diverse expression of prostate cancer-related proteins, this should be recognized in interpreting the immunoprofile of DAC. The diverse expression of mTOR-related proteins implicates their potential utility as predictive markers for mTOR targeted therapy.
\end{abstract}

Key Words: Prostatic neoplasms; Carcinoma, ductal; Immunohistochemistry; Prognosis
Ductal adenocarcinoma (DAC) is an uncommon histologic subtype of prostate cancer, accounting for $3.2 \%$ of prostate cancer cases. ${ }^{1}$ DAC is usually combined with acinar adenocarcinoma (AAC), while its pure form comprises only $0.2 \%-0.4 \%$ of prostate cancers. ${ }^{1}$ DAC is defined by large papillary or cribriform glands lined by tall pseudostratified columnar cells with prominent nucleoli, coarse chromatin, and mitotic figures, which are unusual for AAC. ${ }^{1} \mathrm{DAC}$ is histologically similar to endometrioid adenocarcinoma of the female genital tract, ${ }^{2}$ and thus it was initially described as "endometrial carcinoma of the prostatic utricle" in $1967 .^{3}$ In addition, the histologic features are overlapping with adenocarcinomas of other organs, such as the gastrointestinal tract and lung.

DAC often presents at an advanced stage, frequently with metastasis. ${ }^{4}$ Metastatic spread of DAC occurs commonly in the bone and lymph nodes, similar to AAC. DAC also metastasizes to unusual sites for ACC, such as the lung, liver, and rarely penis, testis, and skin. ${ }^{1,5}$ When DAC presents as a metastatic disease, it poses a diagnostic challenge because of its overlapping features with adenocarcinomas of other organ sites. ${ }^{1}$ In such cases, ancillary studies, like immunohistochemistry, may help make the differential diagnosis. However, the immunoprofile of DAC remains to be defined.

Prostate cancer is dependent on persistent androgen receptor (AR) signaling, which is obtained by overexpression, amplification, point mutations, and splice variants of AR. There are additional signaling pathways implicated in prostate cancer progression, among which the phosphoinositide 3-kinase (PI3K)/AKT/ mammalian target of rapamycin (mTOR) pathway is notable because it is altered in nearly all advanced prostate cancers. ${ }^{6}$ These findings suggest targeting both $\mathrm{AR}$ and $\mathrm{PI} 3 \mathrm{~K} / \mathrm{AKT} / \mathrm{mTOR}$ pathways as a new therapeutic approach in castration-resistant prostate cancer. ${ }^{6}$ However, an immunohistochemical expression of the mTOR signaling pathway in DAC has not yet been reported.

In an effort to expand our understanding of this rare subtype 
of prostate cancer, we examined the clinicopathological features of 61 cases of DAC and their immunoprofiles of prostate cancerrelated and mTOR pathway-related proteins. Specific attention was paid to define prognostic factors for biochemical recurrence (BCR) and potential predictive markers for mTOR inhibitors.

\section{MATERIALS AND METHODS}

\section{Study samples}

This retrospective study initially included 87 cases that underwent radical prostatectomy for clinically localized prostate cancer and were pathologically diagnosed as DAC between January 1995 and December 2015 at Asan Medical Center (Seoul, Republic of Korea). None of these cases were treated with neoadjuvant androgen deprivation therapy. A total of 26 cases were excluded for the following reasons: 16 cases were reassessed as AAC during retrospective review; nine cases were excluded either because the tumor tissue was too small to construct two representative cores of tissue microarray (TMA) or because formalin-fixed paraffin-embedded tissue blocks were unavailable; and one case was excluded because clinical follow-up data was not available. As such, 61 cases of DAC were included in the final analysis.

Patients' clinicopathological information was obtained from electronic medical records and surgical pathology reports. BCR was defined as a serum prostate-specific antigen (PSA) level $\geq 0.2$ $\mathrm{ng} / \mathrm{mL}$ on two consecutive occasions after achieving undetectable PSA following radical prostatectomy. ${ }^{7}$ All pathologic materials were reviewed for diagnostic reassessment according to the 2016 World Health Organization Tumor Classification. ${ }^{1}$ Gleason score (GS) and pathologic tumor stage (pT) were assigned according to the 2015 modified Gleason grading system and the American Joint Committee on Cancer Staging System, seventh edition, respectively. ${ }^{1,8}$ This study was approved by the Institutional Review Board of Asan Medical Center with a waiver of informed consent (2011-0499).

\section{TMA construction}

A TMA construct of 2-mm-diameter cores was generated from the $10 \%$ neutrally buffered formalin-fixed, paraffin-embedded tissue blocks of radical prostatectomy specimens using a tissue microarrayer (Quick-Ray, Unitma Co. Ltd., Seoul, Korea). Two representative cores from different DAC areas were included for each case.

\section{Immunohistochemistry}

Prostate cancer-related proteins analyzed in this study included pan-cytokeratin (PanCK), PSA, AR, enhancer of zeste homolog 2 (EZH2), p53, and ETS-related gene (ERG). Phosphatase and tensin homolog (PTEN), phospho-mammalian target of rapamycin (p-mTOR), phospho-S6 ribosomal protein (pS6), and 14-3-3 sigma protein were included as mTOR pathway-related proteins. Immunohistochemical staining was performed using an automated staining system (BenchMark XT, Ventana Medical Systems, Tucson, AZ, USA). The primary antibodies used in this study, their dilutions, and the subcellular location of each antigen are summarized in Table 1. Nuclei were counterstained with hematoxylin. Representative expression patterns of these proteins are presented in Fig. 1.

The immunohistochemical staining results were assessed in the DAC component only by two pathologists (S.U.J. and A. K.K.), both of whom were blinded to the associated clinicopathological information. The staining intensity of the antibodies was initially scored as negative, weak, moderate, or strong. Cases with moderate to strong intensity were regarded as positive, and then the average percentage of positive cells in all cores was recorded.

\section{Immunohistochemistry on whole section}

To exclude the issues of intratumoral heterogeneity, immunohistochemistry was performed on whole sections of one negative case, one intermediate case, and one positive case for each antibody. In addition, to exclude technical problems, such as poor formalin-fixation of radical prostatectomy specimens, immunohistochemistry for PanCK was performed on whole sections of all PanCK-negative cases.

Table 1. Antibodies used in the study

\begin{tabular}{|c|c|c|c|}
\hline Antibody & Dilution & Company & $\begin{array}{l}\text { Subcellular } \\
\text { location }\end{array}$ \\
\hline PanCK & $1: 400$ & Leica, Newcastle, UK & Cytoplasm \\
\hline PSA & $1: 200$ & Dako Corp., Carpinteria, CA & Cytoplasm \\
\hline$A R$ & $1: 200$ & Cell Marque, Rocklin, CA & Nucleus \\
\hline ERG & $1: 100$ & Epitomics, Burlingame, CA & Nucleus \\
\hline p53 & $1: 1500$ & Dako Corp., Carpinteria, CA & Nucleus \\
\hline EZH2 & $1: 25$ & $\begin{array}{l}\text { Cell Signal Technology, } \\
\text { Beverly, MA }\end{array}$ & Nucleus \\
\hline PTEN & $1: 100$ & $\begin{array}{l}\text { Cell Signal Technology, } \\
\text { Beverly, MA }\end{array}$ & Cytoplasm/nucleus \\
\hline $\mathrm{p}$-mTOR & $1: 100$ & $\begin{array}{l}\text { Cell Signal Technology, } \\
\text { Beverly, MA }\end{array}$ & Cytoplasm \\
\hline pS6 & $1: 100$ & $\begin{array}{l}\text { Cell Signal Technology, } \\
\text { Beverly, MA }\end{array}$ & Cytoplasm \\
\hline 14-3-3 sigma & $1: 200$ & Sigma, St. Louis, MO & Cytoplasm \\
\hline
\end{tabular}

PanCK, pan-cytokeratin; PSA, prostate-specific antigen; AR, androgen receptor; ERG, ETS-related gene; EZH2, enhancer of zeste Homolog2; PTEN, phosphatase and tensin homolog; p-mTOR, phospho-mammalian target of rapamycin; pS6, phospho-S6 ribosomal protein. 


\section{Statistical analysis}

For descriptive statistics and univariate analyses, all continuous data were expressed as mean \pm standard deviation and were com-

Table 2. Clinicopathological features of 61 cases of ductal adenocarcinoma

\begin{tabular}{lc}
\hline Variable & Value \\
\hline Age (yr) & $68.0 \pm 5.6$ \\
Preoperative PSA (ng/mL) & $11.7 \pm 10.3$ \\
Total tumor volume (\%) & $28.5 \pm 21.5$ \\
DAC component (\%) & $48.3 \pm 32.5$ \\
Predominant component & \\
$\quad$ Ductal & $32(52.5)$ \\
Acinar & $29(47.5)$ \\
Predominant DAC pattern & \\
Papillary & $48(78.7)$ \\
Cribriform & $9(14.7)$ \\
PIN-like & $4(6.6)$ \\
Gleason score & \\
7 & $20(32.8)$ \\
8 & $29(47.5)$ \\
9 & $12(19.7)$ \\
Pathologic tumor stage & \\
pT2a-c & $17(27.9)$ \\
pT3a & $29(47.5)$ \\
pT3b & $15(24.6)$ \\
Tertiary grade 5 & $12(19.7)$ \\
Comedonecrosis & $17(27.9)$ \\
Extraprostatic extension & $42(68.9)$ \\
Lymphovascular invasion & $26(42.6)$ \\
Perineural invasion & $52(85.2)$ \\
Positive surgical margin & $41(67.2)$ \\
Seminal vesicle involvement & $15(24.6)$ \\
Lymph node metastasis & $3(4.9)$ \\
Biochemical recurrence & $26(42.6)$ \\
Death & $2(3.3)$ \\
\hline &
\end{tabular}

Values are presented as mean $\pm \mathrm{SD}$ or number $(\%)$.

PSA, prostate-specific antigen; DAC, ductal adenocarcinoma; PIN-like, prostatic intraepithelial neoplasia-like; SD, standard deviation. pared using Student's t tests. The optimal cut-off value of the protein expression was calculated from the receiver operating characteristic (ROC) curve analysis. Categorical data were compared with the chi-square test. BCR was estimated using the Kaplan-Meier method and the resulting curves were compared by log-rank test. In order to minimize the exclusion of variables that are important in this study, all variables with $\mathrm{p}$-values of $<.1$ in the univariate analysis were included in the multivariate analysis, for which the Cox proportional hazards model was used. The overlapping variables were excluded in the multivariate analysis. Independent variables were chosen by the stepwise method. p-values of $<.05$ were considered statistically significant.

\section{RESULTS}

\section{Clinicopathological features of DAC}

The clinicopathological features of the $61 \mathrm{DAC}$ cases are summarized in Table 2. The median age at the time of radical prostatectomy was 68 years (range, 51 to 77 years), with a median preoperative serum PSA level of $11.7 \mathrm{ng} / \mathrm{mL}$ (range, 0.6 to $66.4 \mathrm{ng} / \mathrm{mL}$ ). The mean total tumor volume was $28.5 \%$ (range, $2 \%$ to $95 \%$ ), in which the DAC component occupied $48.3 \%$ on average (range, $5 \%$ to $100 \%$ ). Four cases (6.5\%) were pure DAC. Among histologic DAC patterns, the papillary pattern was the most common (48 cases, $78.7 \%$ ), followed by cribriform pattern (nine cases, $14.7 \%$ ) and prostatic intraepithelial neoplasia-like pattern (four cases, $6.6 \%)$. A significant proportion of the cases were of high grade (GS $\geq 8$ : 41 cases, 67.2\%) with accompanying comedo-necrosis in 17 cases (27.9\%). The majority of the cases were of high stage (pT3: 44 cases, 72.1\%) with frequent extraprostatic extension (42 cases, 68.9\%), lymphovascular invasion (26 cases, $42.6 \%$ ), positive surgical margin (41 cases, 67.2\%), and seminal vesicle
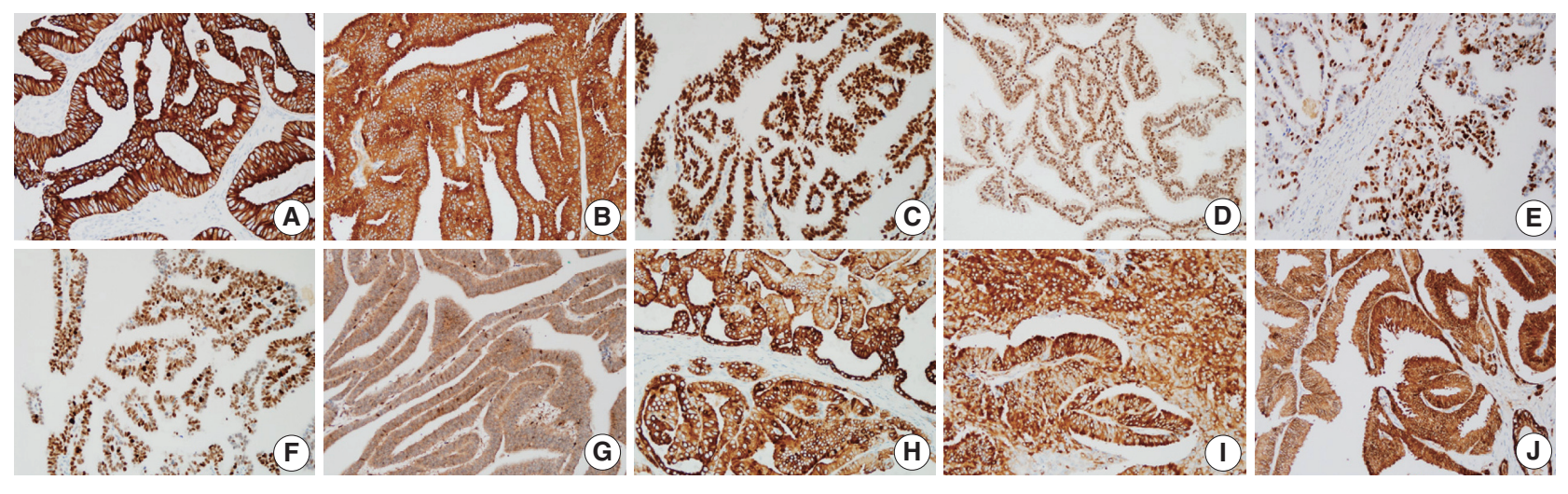

Fig. 1. Representative cases with strong intensity of each immunohistochemical staining: pan-cytokeratin (A), prostate-specific antigen (B), androgen receptor (C), ETS-related gene (D), p53 (E), enhancer of zeste homolog $2(F)$, phosphatase and tensin homolog (G), phosphomammalian target of rapamycin (H), phospho-S6 ribosomal protein (I), and 14-3-3 sigma (J). 


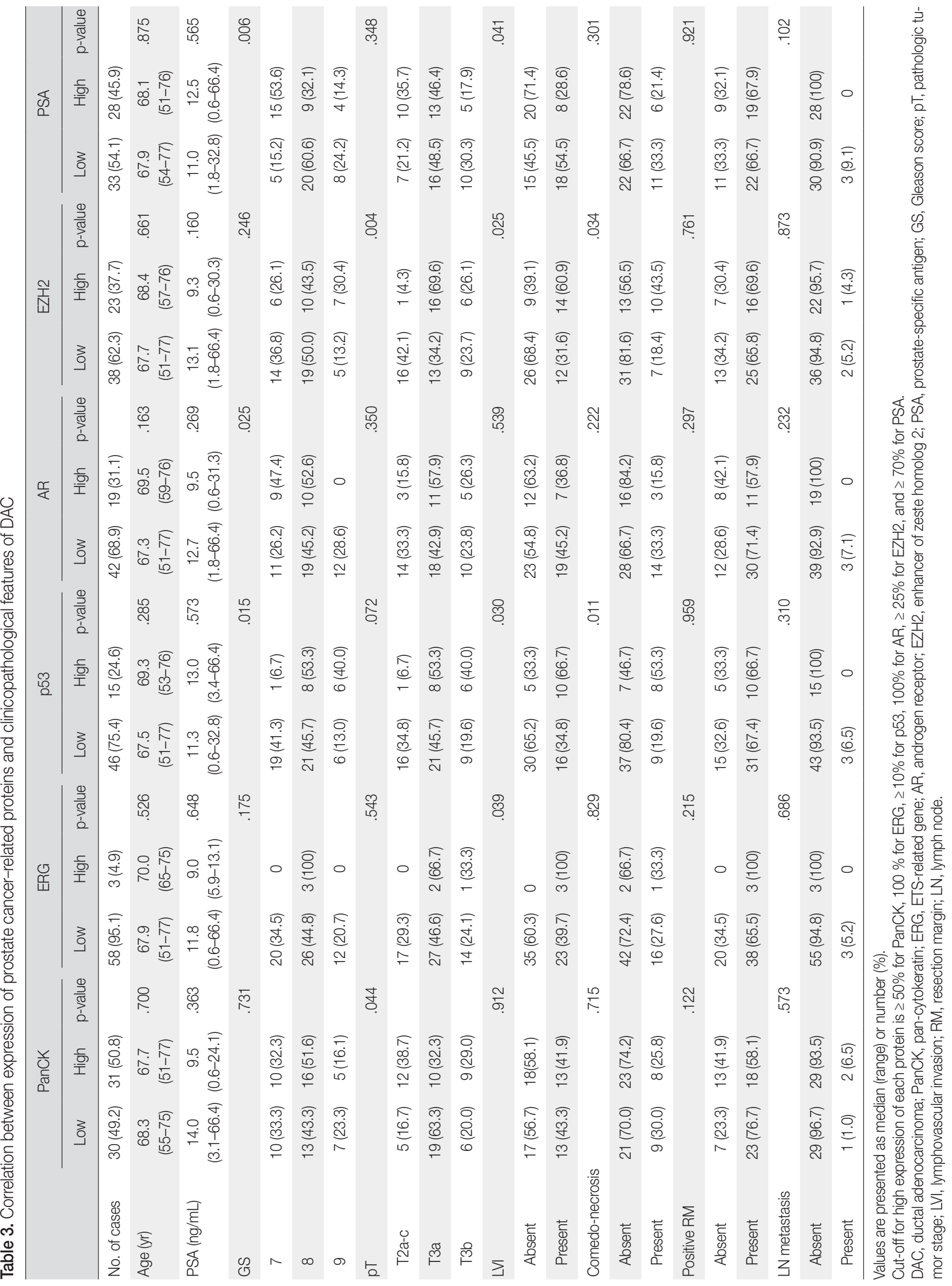


Table 4. Correlation between expression of mTOR signaling-related proteins and clinicopathological features of DAC

\begin{tabular}{|c|c|c|c|c|c|c|c|c|c|c|c|c|}
\hline & & $p-m T O R$ & & & t-3-3 sigma & & & pS6 & & & PTEN & \\
\hline & Low & High & $\overline{p \text {-value }}$ & Intact & Loss & $p$-value & Low & High & $p$-value & Intact & Loss & $p$-value \\
\hline No. of cases & 46 (75.4) & 15 (24.6) & & $25(41.0)$ & $36(59.0)$ & & 39 (63.9) & $22(36.1)$ & & $37(60.7)$ & 24 (39.3) & \\
\hline Age (yr) & $\begin{array}{c}67.8 \\
(51-77)\end{array}$ & $\begin{array}{c}68.7 \\
(59-76)\end{array}$ & .589 & $\begin{array}{c}69.6 \\
(54-77)\end{array}$ & $\begin{array}{c}66.9 \\
(51-76)\end{array}$ & .066 & $\begin{array}{c}66.5 \\
(54-76)\end{array}$ & $\begin{array}{c}68.9 \\
(51-77)\end{array}$ & .109 & $\begin{array}{c}68.7 \\
(59-77)\end{array}$ & $\begin{array}{c}66.8 \\
(51-75)\end{array}$ & .197 \\
\hline PSA (ng/mL) & $\begin{array}{c}11.6 \\
(0.6-66.4)\end{array}$ & $\begin{array}{c}12.0 \\
(3.7-31.3)\end{array}$ & .883 & $\begin{array}{c}14.7 \\
(0.6-66.4)\end{array}$ & $\begin{array}{c}9.6 \\
(1.8-32.8)\end{array}$ & .088 & $\begin{array}{c}14.1 \\
(0.6-66.4)\end{array}$ & $\begin{array}{c}10.3 \\
(0.3-31.3)\end{array}$ & .166 & $\begin{array}{c}13.4 \\
(3.4-66.4)\end{array}$ & $\begin{array}{c}9.1 \\
(0.6-30.3)\end{array}$ & .106 \\
\hline GS & & & .307 & & & .298 & & & .105 & & & .747 \\
\hline 7 & 15 (32.6) & $5(33.3)$ & & $11(44.0)$ & $9(25.0)$ & & $12(30.8)$ & $8(36.4)$ & & $11(29.7)$ & $9(37.5)$ & \\
\hline 8 & $20(43.5)$ & $9(60.0))$ & & $10(40.0)$ & 19 (52.8) & & $22(56.4)$ & $7(31.8)$ & & 19 (51.4) & $10(41.7)$ & \\
\hline 9 & $11(23.9)$ & $1(6.7)$ & & $4(16.0)$ & $8(22.2)$ & & $5(12.8)$ & $7(31.8)$ & & $7(18.9)$ & $5(20.8)$ & \\
\hline pT & & & .138 & & & .426 & & & .790 & & & .051 \\
\hline T2a-c & $12(26.1)$ & $5(33.3)$ & & 8 (32.0) & $9(25.0)$ & & 12 (30.8) & 5 (22.7) & & 8 (21.6) & $9(37.5)$ & \\
\hline Т3a & 25 (54.3) & $4(26.7)$ & & $13(52.0)$ & $16(44.4)$ & & 18 (46.2) & $11(50.0)$ & & 16 (43.2) & $13(54.2)$ & \\
\hline T3b & 9 (19.6) & $6(40.0)$ & & $4(16.0)$ & $11(30.6)$ & & 9 (23.1) & $6(27.3)$ & & $13(35.1)$ & 2 (8.3) & \\
\hline LVI & & & .813 & & & .730 & & & .737 & & & .903 \\
\hline Absent & $26(56.5)$ & $9(60.0)$ & & $15(60.0)$ & 20 (55.6) & & $23(59.0)$ & $12(54.5)$ & & 21 (56.8) & 14 (58.3) & \\
\hline Present & $20(43.5)$ & $6(40.0)$ & & $10(40.0)$ & $16(44.4)$ & & $16(41.0)$ & $10(45.5)$ & & 16 (43.2) & $10(41.7)$ & \\
\hline Comedonecrosis & & & .905 & & & .574 & & & .605 & & & .687 \\
\hline Absent & $33(71.7)$ & $11(73.3)$ & & $19(76.0)$ & $25(69.4)$ & & $29(74.4)$ & 15 (68.2) & & $26(70.3)$ & $18(75.0)$ & \\
\hline Present & $13(28.3)$ & $4(26.7)$ & & $6(24.0)$ & $11(30.6)$ & & $10(25.6)$ & 7 (31.8) & & $11(29.7)$ & $6(25.0)$ & \\
\hline Positive RM & & & .224 & & & .076 & & & .904 & & & .942 \\
\hline Absent & $17(37.0)$ & $3(20.0)$ & & $5(20.0)$ & $15(41.7)$ & & $13(33.3)$ & 7 (31.8) & & $12(32.4)$ & 8 (33.3) & \\
\hline Present & $29(63.0)$ & $12(80.0)$ & & $20(80.0)$ & $21(58.3)$ & & $26(66.7)$ & 15 (68.2) & & $25(67.6)$ & $16(66.7)$ & \\
\hline LN metastasis & & & .310 & & & .782 & & & .919 & & & .320 \\
\hline Absent & 43 (93.5) & $15(100)$ & & $24(96.0)$ & 34 (94.4) & & 37 (94.9) & $21(95.5)$ & & 36 (97.3) & $22(91.7)$ & \\
\hline Present & $3(6.5)$ & 0 & & $1(4.0)$ & $2(5.6)$ & & $2(5.1)$ & $1(4.5)$ & & $1(2.7)$ & $2(8.3)$ & \\
\hline
\end{tabular}

Values are presented as number (\%) or median (range).

Cut-off for each protein is as follows: $\geq 40 \%$ for high expression of p-mTOR, $<80 \%$ for loss of $14-3-3$ sigma, $\geq 10 \%$ for high expression of pS6, and $<85 \%$ for loss of PTEN.

mTOR, mammalian target of rapamycin; DAC, ductal adenocarcinoma; p-mTOR, phospho-mammalian target of rapamycin; pS6, phospho-S6 ribosomal protein; PTEN, phosphatase and tensin homolog; PSA, prostate-specific antigen; GS, Gleason score; pT, pathologic tumor stage; LVI, lymphovascular invasion; $\mathrm{RM}$, resection margin; LN, lymph node.

involvement (15 cases, 24.6\%).

During the median follow-up period of 19.3 months (range, 1 to 70 months), BCR occurred in 26 cases (42.6\%) at a median of 10.5 months (range, 1 to 44 months) after the surgery. Two patients $(3.3 \%)$ died, and one died of prostate cancer (1.6\%).

\section{Expression of prostate cancer-related proteins in DAC}

The prostate cancer-related proteins showed diverse expressions in DAC as shown in Fig. 2A. PanCK and PSA were heterogeneously expressed with a median value of $50 \%$ and $65 \%$, respectively (range, $0 \%$ to $100 \%)$. Furthermore, nine cases (14.8\%) were negative for PanCK and and two cases (3.3\%) for PSA. AR was expressed in all DAC cases with a heterogeneous pattern and a median value of $85 \%$ (range, $10 \%$ to $100 \%$ ). ERG expression was not observed in most cases ( 54 cases, $88.5 \%$ ) and only seven cases $(11.5 \%)$ showed focal or diffuse positivity. $\mathrm{p} 53$ and EZH2 were expressed at median values of $17.5 \%$ (range, $0 \%$ to $35 \%$ ) and $20 \%$ (range, $0 \%$ to $95 \%$ ), respectively.

\section{Expression of mTOR signaling-related proteins in DAC}

The mTOR signaling-related proteins also showed diverse expressions in DAC, as shown in Fig. 2B. DAC cases showed a high expression of PTEN (median, 100\%) and a low expression of p-mTOR (median, 15\%) and pS6 (median, 15\%). Eight cases (13.1\%) showed no immunoreactivity for PTEN. 14-3-3 sigma was also expressed variably with a median value of $60 \%$ (range, $0 \%$ to $100 \%)$.

\section{Immunohistochemistry on whole section}

Tumor heterogeneity was evaluated by immunohistochemistry using whole sections of one negative case, one intermediate case, and one positive case for each antibody. Although there was a slight variation in the cases of intermediate expression, a great degree of similarity was observed in all cases, especially in negative cases and entirely positive cases (data not shown). PanCK immunohistochemistry on whole sections of all PanCK-negative cases on the TMA construct showed immunopositivity in normal 

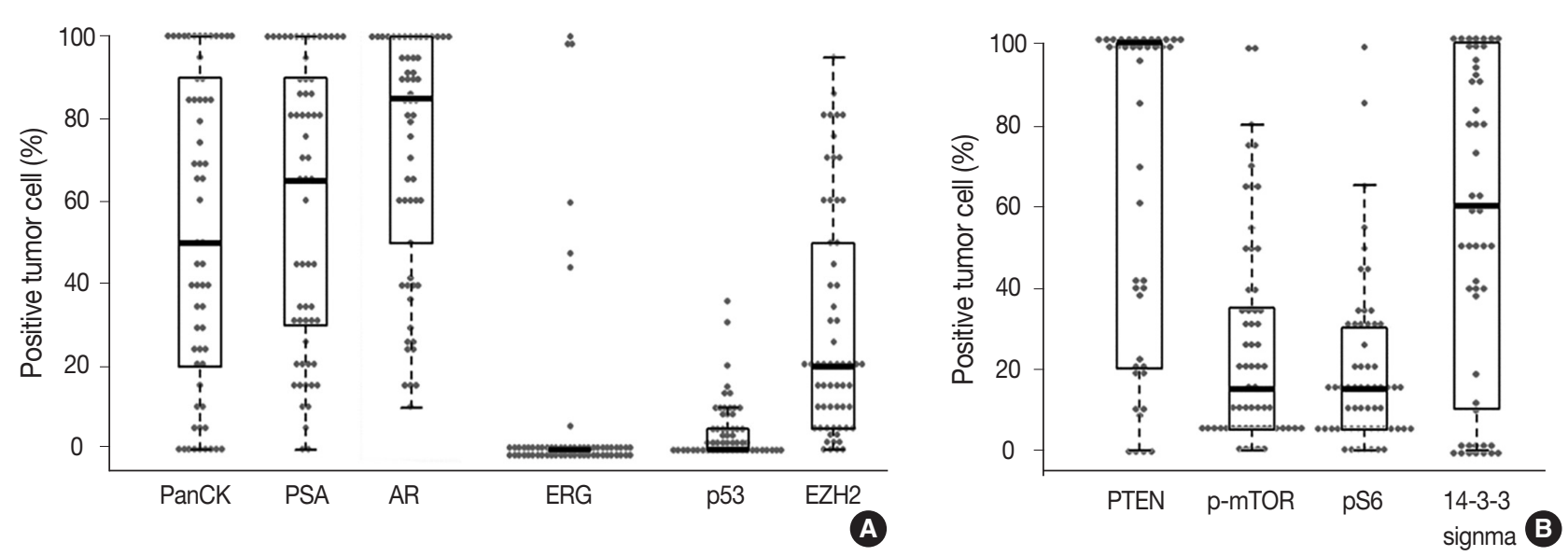

Fig. 2. (A) Box and whisker plot with overlying scatterplot to visualize distributions of the immunoreactive tumor cell proportions against the prostate cancer-related proteins in ductal adenocarcinoma. About $15 \%$ of the cases ( 9 of 61 ) exhibited a completely negative reaction for pan-cytokeratin (PanCK). (B) The same plot to demonstrate the positive tumor cell proportions against the proteins related to the mammalian target of rapamycin (mTOR) pathway. PSA, prostate-specific antigen; AR, androgen receptor; ERG, ETS-related gene; EZH2, enhancer of zeste homolog 2; PTEN, phosphatase and tensin homolog; p-mTOR, phospho-mammalian target of rapamycin; pS6, phospho-S6 ribosomal protein.

prostatic glands and AAC areas as shown in Fig. 3. However, PanCK was negative in eight cases among the nine cases; one case showed focal (15\%) immunopositivity on the whole section. Therefore, technical problems were not an issue and the immunohistochemical data using the TMA construct were confirmed to be representative.

\section{Correlation of protein expression with clinicopathological features}

To define the prognostic significance of the prostate cancerrelated proteins and mTOR signaling-related proteins, a cut-off expression value of each protein was determined according to the ROC curve analysis for BCR (Tables 3, 4). The correlation between expressions of prostate cancer-related proteins or $\mathrm{mTOR}$ signaling-related proteins and clinicopathological features are summarized in Tables 3 and 4, respectively.

Low expression of PanCK was associated with high $\mathrm{pT}$ ( $\mathrm{p}=$ $.044)$, whereas high GS was associated with low expressions of PSA and AR ( $p=.006$ and $p=.025$, respectively) and high expression of $\mathrm{p} 53(\mathrm{p}=.015)$. DAC cases with lymphovascular invasion showed high expressions of ERG, p53, and EZH2 ( $=.039$, p = .030 , and $\mathrm{p}=.025$, respectively) and low expression of PSA ( $\mathrm{p}=$ $.041)$.

\section{Prognostic factors for BCR in DAC}

As shown in Table 5, among the clinicopathological features, the univariate analysis showed that BCR was associated with high preoperative serum PSA level $(\mathrm{p}<.001)$, large tumor volume $(\mathrm{p}<.001)$, predominant ductal component $(\mathrm{p}=.021)$, high GS ( $\mathrm{p}=$ $.004)$, comedo-necrosis ( $\mathrm{p}=0.015)$, high $\mathrm{pT}(\mathrm{p}=.010)$, lymphovascular invasion $(\mathrm{p}=.002)$, and positive surgical margin $(\mathrm{p}=$ .015). Among the protein expressions, high expressions of $\mathrm{p}$ mTOR and low expression of PSA and pS6 were associated with BCR ( $\mathrm{p}=.049, \mathrm{p}=.022$, and $\mathrm{p}=.033$, respectively). Low expression of PanCK showed borderline significance $(\mathrm{p}=.055)$. On multivariable analysis, high GS ( $\mathrm{p}<.001)$, high $\mathrm{pT}(\mathrm{p}=.025)$, low expression of PanCK ( $\mathrm{p}=.007)$, and high expression of $\mathrm{p}$ mTOR ( $\mathrm{p}=.002)$ remained independent prognostic factors for BCR. The Kaplan-Meier survival curves of these four independent prognostic factors are shown in Fig. 4.

\section{DISCUSSION}

Herein, we analyzed the clinicopathological features and immunoprofile of 61 cases of DAC. The results suggest GS, pT stage, and immunohistochemical expressions of PanCK and p-mTOR as independent prognostic factors for BCR. DAC demonstrated wide expression ranges of prostate cancer-related proteins, which should be recognized during interpretation of immunohistochemical results of DAC. Since DAC demonstrated diverse expression of mTOR-related proteins, these results cautiously suggest their potential utility as predictive markers for mTOR-targeted therapy.

Although previous studies regarding the immunohistochemical expression of DAC exist, they analyzed a small number of cases and mostly focused on PSA and a few other prostate cancer-related proteins. ${ }^{9-17}$ Furthermore, they mostly presented the results as 

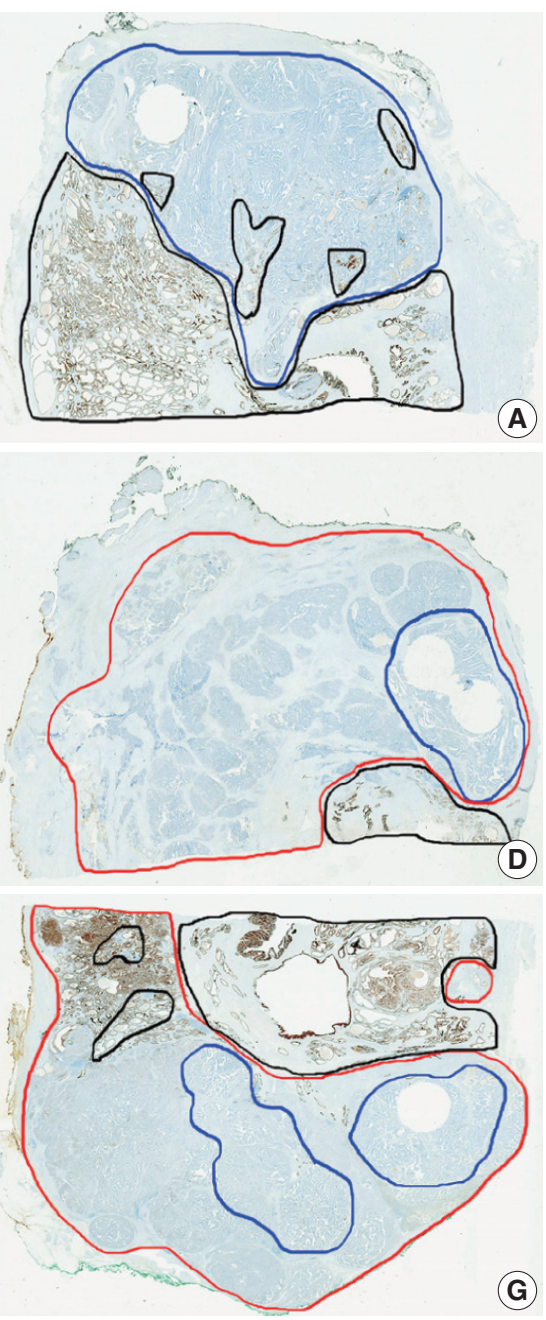
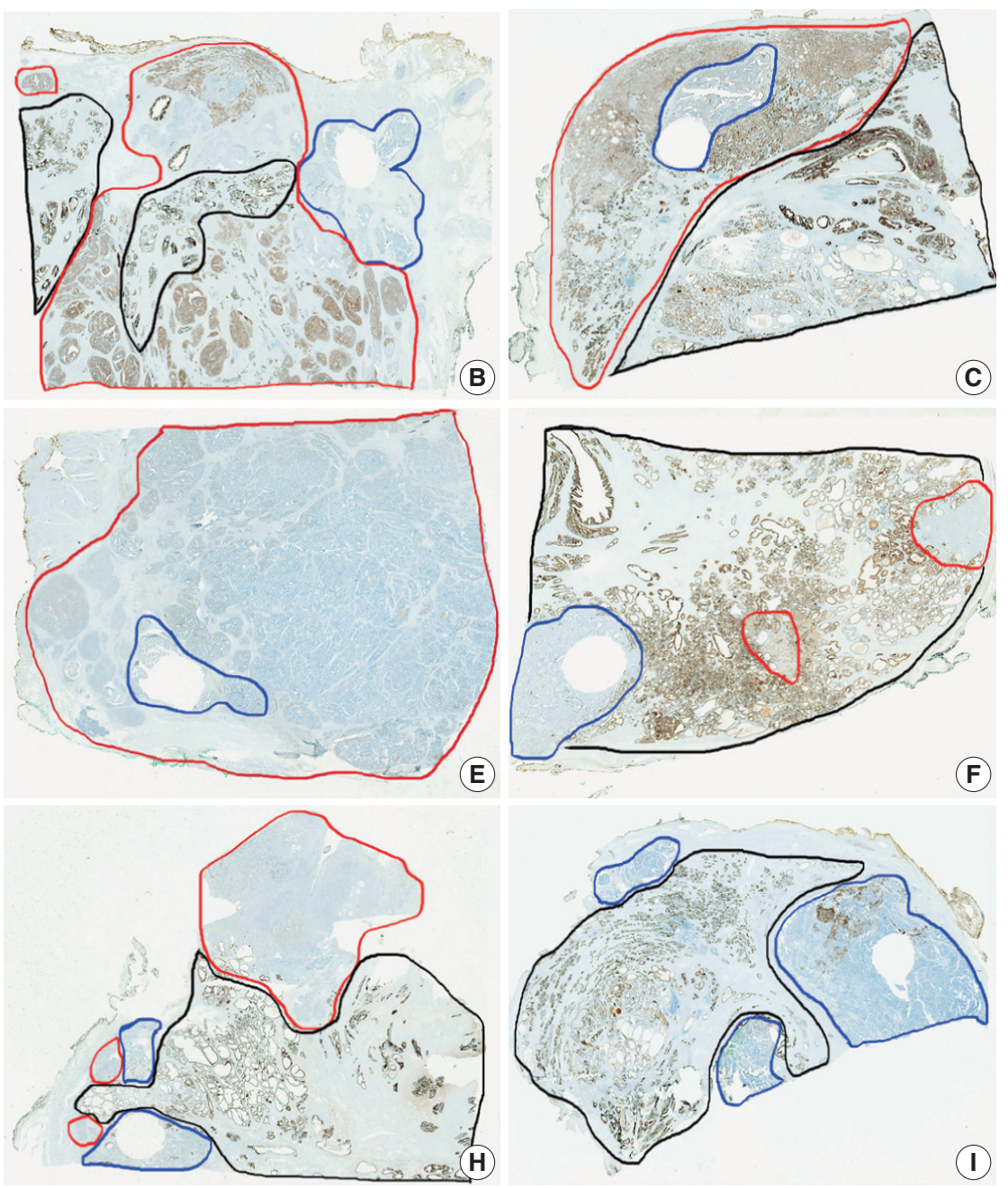

Fig. 3. Pan-cytokeratin (PanCK) immunohistochemistry on whole section slides. All nine cases of PanCK-negative on tissue microarray (TMA) were immunostained for PanCK on whole section, and then their scan view images were presented. PanCK was still negative in eight cases $(\mathrm{A}-\mathrm{H})$ on the whole sections except one case (I), which showed focal (15\%) immunopositivity (ductal adenocarcinoma, blue line; acinar adenocarcinoma component, red line; normal prostate glands, black line; round empty space, TMA site).

positive, focally positive, or negative without an accurate range of expression. One recent study analyzed a large number of cases $(\mathrm{n}=$ 60) and showed high expressions of AR, PSA, and PTEN and low expression of ERG in $100 \%, 100 \%, 70.2 \%$, and $38.3 \%$ of DAC cases, respectively. ${ }^{18}$ Nevertheless, this present study is significant because of the detailed description of the expression range of each protein and the assessment of mTOR pathway-associated protein in DAC for the first time. Furthermore, we identified independent prognostic factors for BCR in DAC: GS, pT, and immunohistochemical expressions of PanCK and p-mTOR.

On light microscopic examination, histologic differences are apparent between DAC and AAC, but it appears that they are similar at the molecular level as assessed by gene expression profile. ${ }^{19}$ In line with this notion, DAC cases in this study showed high expression of AR and low expression of $\mathrm{p} 53$, similar to AAC. ${ }^{20-22}$ PanCK and PSA are drawing special attention among the prostate cancer-related proteins. Even though PanCK and PSA have been proven useful as an epithelial marker and a prostate lineage marker, respectively, the present study showed that they were expressed heterogeneously, including nine cases (14.8\%) of PanCK-negative ones and two cases (3.3\%) of PSA-negative ones. Previous studies showed that $\mathrm{AAC}$ was also focally positive or even negative for PanCK and PSA in a few cases (3.4\% and 2\%-7\%, respectively), similar to DAC in present study. ${ }^{23-27}$ Therefore, it is worth noting that both DAC and AAC could be focally positive and even negative for PanCK and PSA, especially in metastatic disease.

In AAC, fusions between the androgen-regulated transmembrane protease serine 2 gene (TMPRSS2) and the ERG gene are 
Table 5. Univariate and multivariable analyses of the effect of clinicopathological factors and immunohistochemical markers on biochemical recurrence

\begin{tabular}{|c|c|c|c|c|c|c|}
\hline \multirow{2}{*}{ Variable } & \multicolumn{3}{|c|}{ Univariate analysis } & \multicolumn{3}{|c|}{ Multivariable analysis } \\
\hline & $\mathrm{HR}$ & $95 \% \mathrm{Cl}$ & $p$-value & $\mathrm{HR}$ & $95 \% \mathrm{Cl}$ & p-value \\
\hline Age (yr) & 1.010 & $0.941-1.083$ & .783 & - & - & - \\
\hline Preoperative PSA (ng/mL) & 1.066 & $1.038-1.094$ & $<.001$ & - & - & - \\
\hline Total tumor volume (\%) & 1.029 & $1.013-1.047$ & $<.001$ & - & - & - \\
\hline Predominant component (ductal) & 2.793 & $1.171-6.664$ & .021 & - & - & - \\
\hline Gleason score & & & .004 & & & $<.001$ \\
\hline 7 & 1 & & & 1 & & \\
\hline 8 & 12.04 & $1.588-91.21$ & .016 & 15.020 & $1.946-115.941$ & .009 \\
\hline 9 & 26.79 & $3.406-210.66$ & .002 & 26.937 & $3.227-224.851$ & .002 \\
\hline Pathologic tumor stage & & & .010 & & & .025 \\
\hline $\mathrm{T} 2 \mathrm{a}-\mathrm{c}$ & 1 & & & 1 & & \\
\hline ТЗа & 5.400 & $1.205-24.190$ & .028 & 4.270 & $0.890-20.487$ & .070 \\
\hline T3b & 10.190 & $2.237-46.400$ & .003 & 8.288 & $1.617-42.494$ & .011 \\
\hline Comedonecrosis & 2.618 & $1.202-5.702$ & .015 & - & - & - \\
\hline Lymphovascular invasion & 3.728 & $1.615-8.605$ & .002 & - & - & - \\
\hline Positive surgical margin & 4.473 & $1.341-14.930$ & .015 & - & - & - \\
\hline Lymph node metastasis & 0.616 & $0.082-4.624$ & .637 & - & - & - \\
\hline PanCK (high expression) & 0.453 & $0.202-1.016$ & .055 & 0.274 & $0.108-0.700$ & .007 \\
\hline ERG (high expression) & 1.626 & $0.382-6.931$ & .511 & - & - & - \\
\hline p53 (high expression) & 2.082 & $0.938-4.621$ & .072 & - & - & - \\
\hline AR (high expression) & 0.728 & $0.291-1.821$ & .498 & - & - & - \\
\hline EZH2 (high expression) & 2.012 & $0.901-4.490$ & .088 & - & - & - \\
\hline PSA (high expression) & 0.360 & $0.151-0.861$ & .022 & - & - & - \\
\hline p-mTOR (high expression) & 2.266 & $1.004-5.117$ & .049 & 5.184 & $1.829-14.704$ & .002 \\
\hline 14-3-3 sigma (loss of expression) & 1.457 & $0.633-3.356$ & .376 & - & - & - \\
\hline pS6 (high expression) & 0.431 & $0.199-0.935$ & .033 & - & - & - \\
\hline PTEN (loss of expression) & 0.680 & $0.302-1.532$ & .352 & - & - & - \\
\hline
\end{tabular}

$\mathrm{HR}$, hazard ratio estimated by Cox proportional hazards regression model; $\mathrm{Cl}$, confidence interval of the estimated HR; PSA, prostate-specific antigen; PanCK, pan-cytokeratin; ERG, ETS-related gene; AR, androgen receptor; EZH2, enhancer of zeste homolog 2; p-mTOR, phospho-mammalian target of rapamycin; pS6, phospho-S6 ribosomal protein; PTEN, phosphatase and tensin homolog.

present in approximately $40 \%-50 \%$ of cases, where ERG immunohistochemistry correlates well with fusion-positive cancer. ${ }^{24,26}$ In the Korean population, the ERG-positive rate by immunohistochemistry was $24.4 \%$, which is lower than those of Western population-based studies. ${ }^{23,27}$ Interestingly, Japanese populationbased studies also showed low ERG-positive rates, similar to Korean population. These findings suggest that geographic variation may contribute to the lower rates of ERG-positive cases in Eastern Asian prostate cancer patients. ${ }^{27}$ Since only seven cases (11\%) were positive for ERG in the present study, it appears that the ERG-positive rate is even lower in DAC than in AAC.

$\mathrm{EZH} 2$ is the catalytic subunit of the polycomb repressive complex (PRC2) responsible for conducting histone methylation. It is important in cell cycle regulation and has a role in tumor cell proliferation and invasive growth. ${ }^{28}$ High expression of EZH2 in AAC has been associated with aggressive clinicopathological features, such as $G S \geq 8$, extraprostatic extension, positive surgical margins, and BCR. ${ }^{29}$ In contrast to AAC, EZH2 expression was not associated with BCR in DAC by univariate and multivariable analyses, although it was correlated with poor prognostic clinicopathological features, such as comedo-necrosis, high pT, and lymphovascular invasion.

The mTOR pathway responds to diverse environmental cues, such as amino acids, stress, oxygen, energy, and growth factors, and it controls many biologic processes that generate or use large amounts of energy and nutrients. ${ }^{30}$ mTOR signaling impacts most major cellular functions, giving it an important role in regulating basic cellular behaviors, such as cellular growth and proliferation. ${ }^{30}$ Overactivation of mTOR signaling contributes to the initiation and development of many types of cancers, including prostate cancer, suggesting that mTOR inhibitors, such as sirolimus, everolimus, and temsirolimus, might lead to an improved patient survival. ${ }^{31}$ However, the identification of biomarkers that predict which tumors will respond to mTOR inhibitors remains an unmet need. 

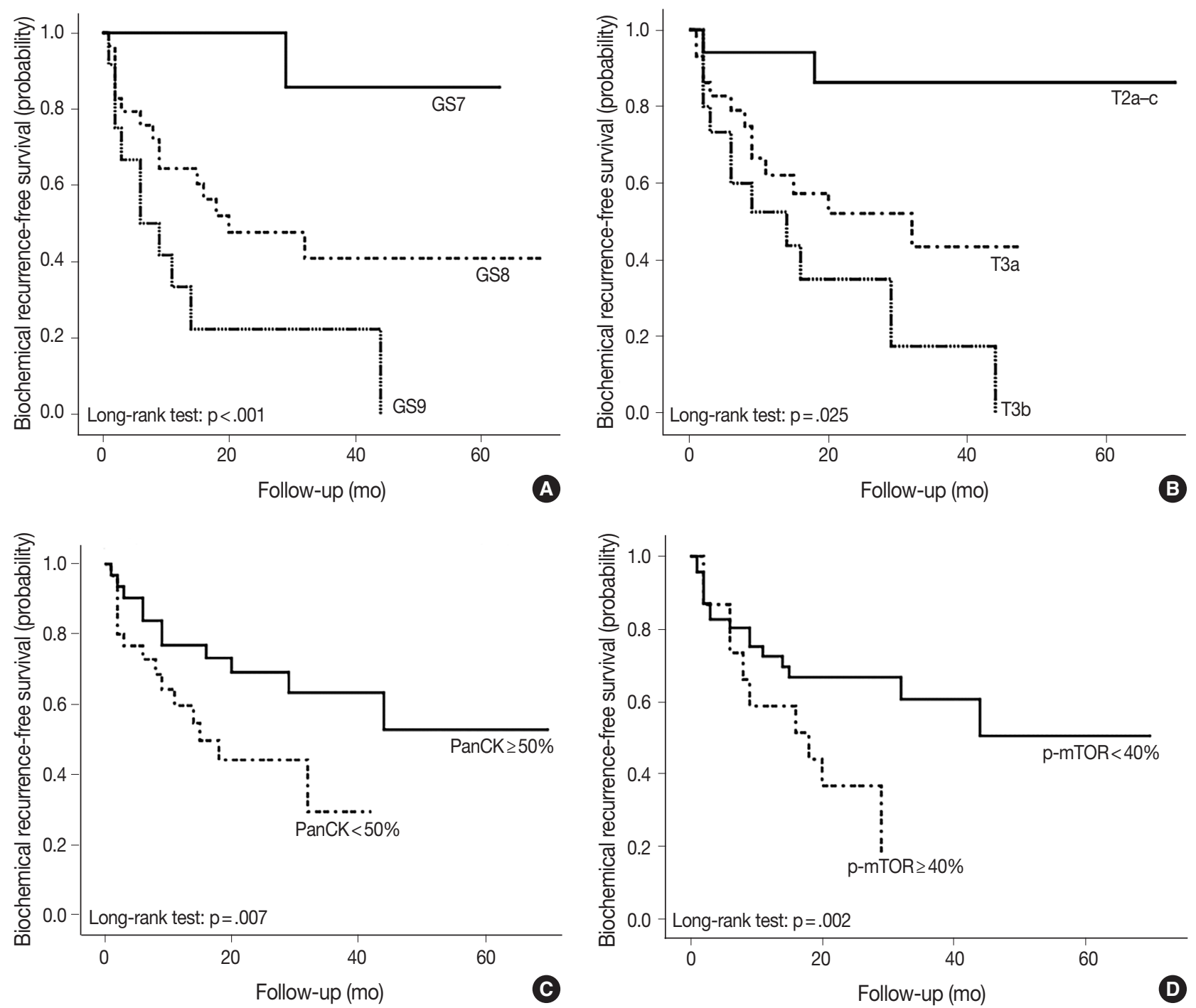

Fig. 4. Kaplan-Meier survival curves of four independent prognostic factors on biochemical recurrence. The Kaplan-Meier survival curves are well-established according to Gleason score (GS) and pT stage (A, B). The prognosis is worse with less than $50 \%$ expression in pan-cytokeratin (PanCK) staining (C), and with more than 40\% expression in phospho-mammalian target of rapamycin (p-mTOR) staining (D).

As one of the diverse upstream regulators of the mTOR pathway, PTEN encodes a phosphatase that dephosphorylates phosphatidylinositol-3,4,5-trisphosphate (PIP3), a second messenger in the $\mathrm{PI} 3 \mathrm{~K}$-protein kinase $\mathrm{B}(\mathrm{PKB})$ signaling pathway. ${ }^{32}$ By negatively regulating the $\mathrm{PI} 3 \mathrm{~K} / \mathrm{PKB}$ signaling pathway, it functions as a tumor suppressor. PTEN loss activates PI3K, which then activates not only mTOR complex 1 (mTORC1) by activating AKT, but also mTORC2 directly. ${ }^{33}$ The mTORC1 and mTORC2 complexes are composed of mTOR and several common and unique proteins, allowing those different sensitivities to upstream regulators and diverse downstream output. ${ }^{30}$ Many stresses, including low energy and oxygen levels and DNA damage, act through tuberous sclerosis 1 (TSC1) and 2 (TSC2), which are key upstream regulators of mTORC1. Adenosine monophosphateactivated protein kinase (AMPK), in response to hypoxia or a low energy state, phosphorylates TSC2 and communicates directly with mTORC1, leading to $14-3-3$ binding. ${ }^{30}$ The binding of 143-3 proteins, including 14-3-3 sigma on mTORC1, may promote mTORC1 signaling under growth factors, but also contributes to the regulatory mechanisms that suppress mTORC1 activity under conditions of cell stress. ${ }^{34}$ mTORC1 also directly phosphorylates and activates ribosomal S6 kinase 1 (S6K1), of which the target substrate is the $\mathrm{S6}$ ribosomal protein (pS6), which has been used as a surrogate for mTORC1 activity. ${ }^{35}$ Phosphorylation of $\mathrm{S} 6$ induces protein synthesis in the ribosome. ${ }^{30}$

Few studies have been conducted on the mTOR signaling 
pathway as a predictive marker in prostate cancer. In a clinical study to evaluate everolimus in castration-resistant prostate cancer, probably AAC type, deletion of PTEN assessed by fluorescence in situ hybridization was found in seven of 23 tumor samples and associated with longer progression-free survival and response. However, they argued that immunohistochemical expressions of PTEN, pS6, p-mTOR, and ERG were not predictive. ${ }^{36}$ To the best of our knowledge, the present study is the first one to assess mTOR pathway-associated proteins in DAC where mTOR-related proteins are diversely expressed. Therefore, it would be interesting to define the usefulness of these proteins as predictive markers of mTOR inhibitors in DAC.

Although our present study examined a relatively large number of DAC cases, it had some limitations, including its retrospective design and the fact that all patients came from a single institution. Most cases were combined with AAC but the AAC component was not evaluated for immunohistochemical expression of prostate cancer- and mTOR signaling-related proteins. Since this present study showed GS, pT stage, and immunohistochemical expressions of PanCK and p-mTOR as independent prognostic factors, multi-institutional studies are necessary to validate the clinical utility of the results. Furthermore, remarkable advances in investigative tools, such as genomic microarray technologies and next-generation sequencing, may help find novel prognostic and predictive biomarkers. Therefore, efforts should be made to identify more accurate markers by integrating newly discovered biomarkers. Although mTOR-related proteins were cautiously suggested as immunohistochemical predictive markers for mTOR inhibitors, this result should be confirmed by immunohistochemical staining on whole section. It is also obvious that the assumption is still premature and should be investigated through a prospective clinical study.

\section{Conflicts of Interest}

No potential conflict of interest relevant to this article was reported.

\section{Acknowledgments}

This research was supported by the Basic Science Research Program through the National Research Foundation of Korea (NRF) funded by the Ministry of Science, ICT and Future Planning (2015R1A2A2A01006958).

\section{REFERENCES}

1. Moch H, Humphrey PA, Ulbright TM, Reuter VE. WHO classification of tumours of the urinary system and male genital organs. 4th ed. Lyon: International Agency for Research on Cancer, 2016.

2. Melicow MM, Pachter M. Endometrial carcinoma of proxtatic utricle (uterus masculinus). Cancer 1967; 20: 1715-22.

3. Tarjan M, Lenngren A, Hellberg D, Tot T. Immunohistochemical verification of ductal differentiation in prostate cancer. APMIS 2012; 120: 510-8.

4. Meeks JJ, Zhao LC, Cashy J, Kundu S. Incidence and outcomes of ductal carcinoma of the prostate in the USA: analysis of data from the Surveillance, Epidemiology, and End Results program. BJU Int 2012; 109: 831-4.

5. Tu SM, Lopez A, Leibovici D, et al. Ductal adenocarcinoma of the prostate: clinical features and implications after local therapy. Cancer 2009; $115: 2872-80$

6. Statz CM, Patterson SE, Mockus SM. mTOR inhibitors in castrationresistant prostate cancer: a systematic review. Target Oncol 2017; 12: 47-59.

7. Jung WY, Sung CO, Han SH, et al. AZGP-1 immunohistochemical marker in prostate cancer: potential predictive marker of biochemical recurrence in post radical prostatectomy specimens. Appl Immunohistochem Mol Morphol 2014; 22: 652-7.

8. Edge SB, Compton CC. The American Joint Committee on Cancer: the 7th edition of the AJCC cancer staging manual and the future of TNM. Ann Surg Oncol 2010; 17: 1471-4.

9. Bostwick DG, Kindrachuk RW, Rouse RV. Prostatic adenocarcinoma with endometrioid features: clinical, pathologic, and ultrastructural findings. Am J Surg Pathol 1985; 9: 595-609.

10. Ro JY, Ayala AG, Wishnow KI, Ordóñez NG. Prostatic duct adenocarcinoma with endometrioid features: immunohistochemical and electron microscopic study. Semin Diagn Pathol 1988; 5: 301-11.

11. Gong Y, Caraway N, Stewart J, Staerkel G. Metastatic ductal adenocarcinoma of the prostate: cytologic features and clinical findings. Am J Clin Pathol 2006; 126: 302-9.

12. Leite KR, Mitteldorf CA, Srougi M, et al. Cdx2, cytokeratin 20, thyroid transcription factor 1 , and prostate-specific antigen expression in unusual subtypes of prostate cancer. Ann Diagn Pathol 2008; 12: 260-6.

13. Copeland JN, Amin MB, Humphrey PA, Tamboli P, Ro JY, Gal AA. The morphologic spectrum of metastatic prostatic adenocarcinoma to the lung: special emphasis on histologic features overlapping with other pulmonary neoplasms. Am J Clin Pathol 2002; 117: 552-7.

14. Oxley JD, Abbott CD, Gillatt DA, MacIver AG. Ductal carcinomas of the prostate: a clinicopathological and immunohistochemical 
study. Br J Urol 1998; 81: 109-15.

15. Lee SS. Endometrioid adenocarcinoma of the prostate: a clinicopathologic and immunohistochemical study. J Surg Oncol 1994; 55: 235-8.

16. Millar EK, Sharma NK, Lessells AM. Ductal (endometrioid) adenocarcinoma of the prostate: a clinicopathological study of 16 cases. Histopathology 1996; 29: 11-9.

17. Tulunay O, Orhan D, Baltaci S, Gögüş C, Müftüoglu YZ. Prostatic ductal adenocarcinoma showing Bcl-2 expression. Int J Urol 2004; 11: 805-8.

18. Seipel AH, Samaratunga H, Delahunt B, Wiklund P, Clements M, Egevad L. Immunohistochemistry of ductal adenocarcinoma of the prostate and adenocarcinomas of non-prostatic origin: a comparative study. APMIS 2016; 124: 263-70.

19. Sanati S, Watson MA, Salavaggione AL, Humphrey PA. Gene expression profiles of ductal versus acinar adenocarcinoma of the prostate. Mod Pathol 2009; 22: 1273-9.

20. Ruizeveld de Winter JA, Janssen PJ, Sleddens HM, et al. Androgen receptor status in localized and locally progressive hormone refractory human prostate cancer. Am J Pathol 1994; 144: 735-46.

21. Henshall SM, Quinn DI, Lee CS, et al. Altered expression of androgen receptor in the malignant epithelium and adjacent stroma is associated with early relapse in prostate cancer. Cancer Res 2001; 61: 423-7.

22. Schlomm T, Iwers L, Kirstein P, et al. Clinical significance of p53 alterations in surgically treated prostate cancers. Mod Pathol 2008; 21: 1371-8.

23. Lee K, Chae JY, Kwak C, Ku JH, Moon KC. TMPRSS2-ERG gene fusion and clinicopathologic characteristics of Korean prostate cancer patients. Urology 2010; 76: 1268.e7-13.

24. Epstein JI, Egevad L, Humphrey PA, Montironi R, Members of the IIIDUPG. Best practices recommendations in the application of immunohistochemistry in the prostate: report from the International Society of Urologic Pathology consensus conference. Am J Surg Pathol 2014; 38: e6-19.

25. Berner A, Harvei S, Tretli S, Fosså SD, Nesland JM. Prostatic carci- noma: a multivariate analysis of prognostic factors. Br J Cancer 1994; 69: 924-30.

26. Morais CL, Herawi M, Toubaji A, et al. PTEN loss and ERG protein expression are infrequent in prostatic ductal adenocarcinomas and concurrent acinar carcinomas. Prostate 2015; 75: 1610-9.

27. Suh JH, Park JW, Lee C, Moon KC. ERG immunohistochemistry and clinicopathologic characteristics in Korean prostate adenocarcinoma patients. Korean J Pathol 2012; 46: 423-8.

28. Bachmann IM, Halvorsen OJ, Collett $\mathrm{K}$, et al. EZH2 expression is associated with high proliferation rate and aggressive tumor subgroups in cutaneous melanoma and cancers of the endometrium, prostate, and breast. J Clin Oncol 2006; 24: 268-73.

29. van Leenders GJ, Dukers D, Hessels D, et al. Polycomb-group oncogenes EZH2, BMI1, and RING1 are overexpressed in prostate cancer with adverse pathologic and clinical features. Eur Urol 2007; 52: 455-63.

30. Laplante M, Sabatini DM. mTOR signaling in growth control and disease. Cell 2012; 149: 274-93.

31. Xu K, Liu P, Wei W. mTOR signaling in tumorigenesis. Biochim Biophys Acta 2014; 1846: 638-54.

32. Giannico GA, Arnold SA, Gellert LL, New and emerging diagnostic and prognostic immunohistochemical biomarkers in prostate pathology. Adv Anat Pathol 2017; 24: 35-44.

33. Sabatini DM. mTOR and cancer: insights into a complex relationship. Nat Rev Cancer 2006; 6: 729-34.

34. Morrison DK. The 14-3-3 proteins: integrators of diverse signaling cues that impact cell fate and cancer development. Trends Cell Biol 2009; 19: 16-23.

35. Templeton AJ, Dutoit V, Cathomas R, et al. Phase 2 trial of singleagent everolimus in chemotherapy-naive patients with castrationresistant prostate cancer (SAKK 08/08). Eur Urol 2013; 64: 150-8.

36. Vaishampayan U, Shevrin D, Stein M, et al. Phase II trial of carboplatin, everolimus, and prednisone in metastatic castration-resistant prostate cancer pretreated with docetaxel chemotherapy: a prostate cancer clinical trial consortium study. Urology 2015; 86: 1206-11. 